\section{ON THE TREATMENT OF PLEURITIC EFFUSION.*}

By T. CLIFFORD ALLBUTT, M.A., M.D., Physician to the General Infirmary, Leeds.

IN the paper I am to read before this Section, I can scarcely hope to say much that is new, but I may hope to put the old before you strengthened by the results of a maturer experience. My own views of the treatment of pleuritic $\epsilon$ ffusion have been laid repeatedly before the profession, and have contributed to the formation of a bolder habit of procedure in the matter. It is, therefore, neither needful nor possible in the time before me to enter into any full or minute discussion of the whole of our subject, but rather to set forth as pointedly as possible my views on the more difficult or disputed points. Under such circumstances, I must ask your forgiveness for any apparent abruptness or degmatism.

It appears to me that our first duty is so to divide pleurisies into classes as to enable us to know more clearly what we have to deal with, and thus to avoid much of that controversy which gathers about ill-defilcd propositions as parasites gather about ill-nourished tissues. Althongh no hard lines can be drawn around them, yet the following divisions are fairly recognisable if we disregard transitional cases.

I. Dry pleurisies, in which the tubercular may be included.

2. Acute effusive pleurisies, in which the rheumatic are included.

3. Quiet effusive pleurisies in the serous stage.

4. Empyemata.

5. Pleuritic dropsy.

Of Class I, I have now nothing to say. The tubercular pleurisies are at times effusive, but the exigencies of individual cases are too vat ious to be here considered.

Class II. Acute effusive pleurisies are those of an actively inflam. ma: ory kind, which make themselves sharply felt from the beginning by pyrexia and pain. The treatment of such cases seems to me to be clear. It is this. At the outset, that is, within twenty-four or fortyei ${ }_{\text {. }}$ t hours at farthest, leeches should be liberally applied to the parts, according to the forces of the patient, and a poultice applied to receive the bleeding. As soon as the bleeding has ceased, the affected side should be bound down by strapping after the manner best described by Dr. Roberts. Of medicines, I advise a mild saline purgative at the beginning, followed by the use of mercury and chalk combined with Dover's powder in fractional doses, or in weakly patients by the use of Dover's powder alone. Between these powders, I give a mixture containing acetate of potash and large doses of liquor ammoniæ acetatis. By this method, I obtain far better results than were wont to follow my expectant treatment of former years. The fibrinous effusion which issues in these cases almost always subsides when it has reached its height ; and, if this height be the height of the spine of the scapula and the fourth rib, I am for this reason never in haste to interfere by operation so long as the patient breathes in tolerable comfort and the other lung is well at work. On the other hand, if the patient be uneasy, or if the entry of blood to the right heart be hindered, I do not hesitate to tap at once. The favourable aspect of operation in such cases is that suppurative conversion rarely occurs in these highly organised effusions, even if air enter the pleura. Once, in a case of impending death from double rheumatic pleurisy and pericarditis, all with effusion, we, being in haste, did not hesitate to plunge a bistoury into the fuller pleural cavity, and to allow the effusion to escape as it might, relying on the highly fibrinous quality of it which did not tend to suppuration. The wound soon closed, and the patient did well. Such effusions, being full of clots, are often difficult to remove by small cannulæ, or even to exhaust by aspiration ; if opportunity permit, however, the proper plan is to use the aspirator with fine cantulæ, and to puncture the pleura repeatedly, drawing off what is to be had at each point. It is better to do this than to fumble in the first puncture, and the patient and his friends must be prepared beforehand for the probability of repeated punctures ; I repeat, however, that operation is rarely needed in acute f.brinous pleurisy, and that its exudations, even if profuse, tend for the must to steady reabsorption. If, when all fever is past, such an effusion J nger at its height or linger after a partial ebb, the use of a blister or $r t$ peated blisters certainly favours its removal. It is better to repeat the blisters than to allow the first or any one of them to proceed to full $v e$ ication. Scmetimes, indeed, these measures may fail, and abiding dulness, silence, and immobility in the affected side will continue. Such a condition is often treated with indifference, and no doubt some

* Read in the Section of Medicine at the Annual Meeting of the British Medical A: sociation in Manchester, August 1877 . time may elapse before such a side is completely restored to its normal state ; nay, more, it is rare that the marks of such a pleurisy vanish as they came. More often they remain for years, or even for a lifetime. But, on the other hand, if the dulness and other signs be considerable, I am very unwilling to treat them with neglect, for such conditions may end in serious impairment of the lung, and even to chronic interstitial fibrosis of the lung. It is my practice, therefore, and I speak from some experience, as pleurisy is very common in Yorkshire, to put the patient under a course of mercury rather than allow this morbid state to remain. A combination of the bichloride of mercury with iodide of potassium and bark or iron may be given fearlessly for weeks, and will rarely fail to promote the removal of the remaining products of the inflammation and to restore health and activity to the affected organs. Such a course must needs be given most carefully, and the patient, on the conclusion of it, advised to take sea-air and tonic medicines. Routine drugging, pursued in ignorance of the natural course of disease, very rightly was displaced by expectant treatment ; yet I fear that expectant treatment, having now helped us to learn more clearly the ways of disease, has in its turn sins of omission to answer for as great as or greater than the sins of commission laid at the door of the apothecary.

I will now pass on to Class III, quiet effusive pleurisy in the serous stage. Although the acuter pleurisies may run to large effusions and to effusions poor in fibrin, yet we more commonly see the larger and poorer effusions in cases where the pain has been trifling and the pyrexia moderate if more continuous. A daily evening rise of two degrees is easily overlooked by the physician and easily regarded by the patient as mere malaise. Such patients, with one moiety of the chest full or nearly full of water, are treated with tonics to relieve debility and anæmia, or are sent to watering-places to recruit their strength, until, perhaps, their actual state is revealed by accident. If such effusions come on slowly, as no doubt they often do, the sufferer may complain of but little more dyspnœa than is common to most weakly persons, and one patient who consulted me was able to lie on either side and to sleep on either side, although his left pleura was crammed with effusion. On the other hand, such effusions may come on with great rapidity and destroy life by the sudden dislocation of parts. Such cases, however, are not likely to be misapprehended; as, although fever and pain may be slight or absent, the dyspnœa compels a minute examination of the chest. But, let me earnestly impress upon my brethren a warning which, sounded again and again, has not yet aroused the profession to a full sense of the perilous state of those whose chests contain large effusions. Where the water floods the chest in a few days or hours, the alarm may be taken, it can scarcely be neglected; but those whose effusions have gathered more stealthily are in as great a danger if time pass and no precautions be taken. One terrible warning in my earlier life taught me this lesson, while it turned my heart to the search for help to these sufferers. When yet upon the threshold of my medical studies, I was standing on the terrace in front of Addenbrooke's Hospital waiting for a young girl who had descended from a market-cart and was walking, slowly it may be but firmly, towards the house. She had crossed the green, when suddenly a cry escaped her and she fell dead at my feet. The porter and myself raised her, and gave restoratives in vain. She was gone, and the cause of her premature and sudden end was the effusion of fluid into the left pleural cavity. Now, shocking as this was to one who saw it, it is by no means an uncommon accident. At least thrice in my experience at the Leeds Infirmary have patients thus fallen dead from the same cause, and some instances of the like have come before me in my private practice. Let him, then, who hesitates to tap the pleura remember that, before his next visit, his patient, seemingly so tranquil, may have passed into the deeper stillness of death. Whether the effusion, then, be rapid or be slow in its flood, if the cavity be full, operate without delay. This is, I believe, one of those golden rules to which there is no exception. If the effusion be below the capacity of the pleura, immediate action is less imperative. Speaking of myself alone, I have never seen death by syncope except from a full pleura, though I presume such a death is possible. It depends, no doubt, on a dislocation of the heart and great veins, such as to form clot, or directly to impede the filling of the auricle or auricles; and I believe such pressure is rarely exerted to any degree until the compression of the lung has reached its limit. Still, I shrink, even before a patient at his ease, from allowing the breadth of three fingers to stand between him and death. A sudden swelling of the tide might occur even in the night, and help be absent. Moreover, the continued pressure of such exudation by soddening injures the lung, or by extending adhesions favours the permanent imprisonment of this organ, or by its own deterioration drifts towards an empyema. Nevertheless, with a patient of good promise, with fairly full arteries and respirations under 30 , and whose exudation reaches no higher than 
the scapular ridge behind and the third rib in front, I counsel delay, warning the patient against rising up suddenly, and instructing his attendant to call the doctor in case of more numerous breathing or a change of complexion. If the patient be able to take solids, I advise a dry diet, gentle saline purgatives such as Hunyadi water, and syrup of iodide of iron with digitalis. Mercury I withhold, save as an occasional alterative. I do not strap the chest, as I prefer to be able to apply repeated blisters, stopping short of vesication. These quiet effusions are, however, hard to move, and so often increase that one is not sorry to have to operate and thus to shorten the duration of the case. In my inmost heart, I believe it will be found better in the end to tap all cases where more than two pints of fluid are present, as the results of medicine alone in quiet effusions are very tedious and unsatisfactory. An operation upon the chest is, however, as yet too unfamiliar and too dreudiul to the public to permit us to turn to it hastily, and in these cases there is the not inconsiderable risk of so setting up an empyema, a risk nearly absent in mere fibrinous effusions on the one hand, and in mere dropsies on the other. It can scarcely be doubted, however, that tapping of the pleura, as it becomes better known and the procedure more perfect, will be applied to those smaller effusions which persist in spite of a short course of nursing and medicine.

In now confining myself to the larger effusions, let me again repeat, formally and unmistakably, that physicians must admit that the medicinal treatment of the larger quiet effusions is, on the whole, a failure, and where it succeeds runs the risk of injury to the lung, of empyema, and even of sudden death. Not only so, but pleuritic effusions in the right cavity, by pressing upon the vena cava and twisting it upon the heart, are not uncommonly attended by dropsy in the legs and elsewhere. On the other hand, my own experience of operation in large serous effusion is very favourable; and, if I confine myself to simple cases and early operation, my results have been excellent, and have converted a seriuus malady into a moderate indisposition. Over and over again, by this procedure is fluid removed in bulk from the chest by one operation nceding no repetition, and rapid recovery is obtained.

In opposiition to sume writers, I find that the chances against reaccumulation are in cases of early operation very moderate and even small ; and, unless pus be formed, a third operation is in my experience quite rare. Add to this, that an illness of three months is reduced to an illness of three weeks, and the merits of early operation are even more convincing. The longer, however, operation is deferred, the less confidently can the best results be hoped for, the more danger of formation of clots and of empyema, and the more the danger of injury to lung and constitution.

The aspirator, which is valuable in highly fibrinous effusion, is even undesirable in serous effusion. It is better to allow the lung to expand at is own pace, and not to draw off more fluid than the lung can at that time replace. Even a partial relief of this kind generally leads to absorption of the remuant, and does not lead to severe cough and albuminous expectoration. Nor do I like instruments with angles in them, which are liable to become clogged. A fine trocar and cannula, the latter attached to a long flexible tube, through the wall of which the trocar should be passed on the distal side of the shoulder, is the best instrument. The tube closes upon the trocar as it is withdrawn, and no air can pass beside or after it, if carefully managed and the trocar be twoedged. A bayonet-pointed trocar wounds the tube too much. The instrument should be well carbolised before insertion, ihe tube filled with carbolised water, and the end immersed in a basin of carbolised water. By raising or loxering the basin, the syphon action may be increased or diminished at will. It is as well to keep a spray in motion about the puncture until all be over and the orifice closed with antiseptic dressing.

Now, of this simple operation our Yorkshire experience is so large that I may permit myself to marvel at the fear or hesitation which it excites, even in the medical breast, and, moreover, to doubt the reality of those untoward consequences which are said at times to follow it. That, if the operation be long deferred, its success is less sure, needs no reiteration; that a person in whom syncope is imminent may not always avert that syncope by operation, especially if the fluid be aspirated rapidly, is possible; that a tendency to clot, or the establishment of clot, in the central blood-vessels, is always to be feared in long standing cases; that a patient honeycombed by disease may die coincidently with or even consequently upon the smallest operation is certain, but who is to be deterred by these events from taking the course of operation in a promising case? In a paper like the present, we cannot discuss rare exceptions ; we can only lay down general rules.

I will now pass on to Class IV-empyema. With empyema, operation of some kind is inevitable, and as, encysted empyemata apart, a pus containing pleura means a full pleura, and as again we have decided that all full pleuræe are to be tapped promptly, there can be no difficulty in the matter of diagnosis. The presence of œdema in the wall of the thorax, however, will generally tell when the contents are purulent. That an encysted empyema may dry up is possible; but, if it do, it leaves caseous matter behind which may become a source of general poisoning-tuberculous or other-or it may remain latent for years and finally cause death, as in the case of a patient who died lately under my observation. In his case, the necropsy showed that an encysted empyema of ten years' standing was the cause of death by perforation of lung, etc., although apparent complete recovery had taken place at the time of the original illness. If I have one conviction in medicine more urgent than another, it is this : if pus or other septic material be present in the body, we must not rest until it is removed. I, therefore, dislike and reprobate all temporising with an empyema. Out with it, and provide against the chance of reaccumulation. We are advised by some persons to draw off an empyema by repeated aspirations.* I have seen two successful cases, so-called, of this practice. One spat pus within a month of his recovery, and the other died of hectic. Twice I have seen pus spat up while the systematic aspirations were carried out. I would not trust any reputed recovery under this plan till the patient had been watched for years. My two objections, and these complete ones, to repeated aspiration are : 1. Aspiration does not prevent the formation of a pulmonary fistula ; 2 . It does not prevent absorption, but rather favours it. By the pressure of a full cavity, absorption is often prevented and fever absent ; draw off some of the pus, you relieve pressure, and absorption begins. I put before you charts illustrative of this ; from them, you will see that by complete drainage alone fever is averted, and by the presence of fever after operation for empyema I detect at once a defect in the operation or a defect in the nursing. By a free opening at the lowest point of the cavity and as far back as possible, the pus must be run out, and complete drainage secured. The whole must be done under the antiseptic method, and antiseptic dressing continued. I have rarely found injections of any value, and should only resort to them in case of very fetid retained material, and should then use them of the mildest and simplest kind. But this plan, it is said, means a three months' illness, and perhaps death by exhaustion. $\mathrm{Be}$ it so, and be it remembered that empyema taking its own course is a three years' illness, and death probably at the end of that. Nor can any sleight of hand make a huge internal abscess anything but a terribie infliction. I can only say that the miserable broken down creatures who seek shelter in a hospital are generally cured completely in twelve or fifteen weeks; and that in private practice I have never yet lost a reasonably favourable case. As the cavity contracts to small dimensions, the drainage-tube must be shortened. If a pulmonary fistula have formed before the patient comes under treatment, a counter-opening must be made and the chest drained, as in other cases.

Class v. Pleural dropsies will not detain us long. These being often dependent upon disease elsewhere, and, therefure, often double, puncture should be used only in case of urgency. Fortunately, in these cases operation is of the simplest kind. There is no fear whatever of pus-formation, and the water may be allowed to run through au unguarded trocar, as in the case of ascites. At the same time, if circumstances permit, it is well to form an antiseptic atmosphere around the opening.

\section{O N P LEURISY OF T H E APEX.†}

\section{BY I. BURNEY YEO, M.D., F.R.C.P.,}

Physician to King's College-Hospital, and Assistant-Physician to the Brompton Hospital for Consumption.

I wisir to call attention to the clinical aspects and to the treatment of a class of cases which, if they have not been altogether overlouked, have at any rate received but scant attention at the hands of physicians, and find no place, that $I$ am aware of, in our medical literature. I allude to cases of plcuritis attacking the apex of one or othtr lung, and attended with characteristic symptomatic phenomena. I need not say that I am making no reference wha: ever to the exceedingly common, nay almost constant, circumstance of the coexistence of affections of the pleura with phihisical consolidation of the upper part of the lung. It is by no means an unusual circumstance to find, in autopsies of persons dying of other than lung-disease, firm adhesions between the layers of pleura covering the apices of the lung, without the existence of any consolidation of those parts. Such persons must

* I mean here aspirations as the chest refills. On the other hand, the more receut proposal to aspirate every other day, or at very short intervals, is, in my opinion, well deserving of careful trial.

$t$ Read in the Section of Medicine at the Annual Meeting of the British Medical Association in Manchester, August 1877 . 